\title{
A Novel Cause-Effect Variable Analysis in Enterprise Architecture by Fuzzy Logic Techniques
}

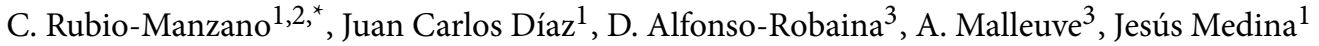 \\ ${ }^{1}$ Department of Mathematics, University Cádiz, Cádiz, Spain \\ ${ }^{2}$ Department of Information Systems, University of the Bio-Bio, Concepción, Chile \\ ${ }^{3}$ Universidad Tecnológica de la Habana CUJAE, La Habana, Cuba
}

\section{ARTICLE INFO}

\section{Article History}

Received 14 Nov 2019

Accepted 07 Apr 2020

\section{Keywords}

Decision-making

Formal analysis of rules

Enterprise architecture

Fuzzy relation equations

Fuzzy sets

\begin{abstract}
In this paper, we present a new integration approach for managing Information Technology variables within enterprise architecture in an integrated way. Additionially, a novel method based on fuzzy logic for cause-effect variable analysis is proposed as a useful support decision-making tool for companies in order to know the main actions they must perform for increasing their benefits. This is employed to assess the Integration Management System in Enterprises, based on Enterprise Architecture and Information Technology. We show as fuzzy logic plays an important role in this area due to these variables can be affected for multifactorial elements impregnated with uncertainty. The knowledge given by the experts is translated into dependence rules, which have also been analyzed from a fuzzy point of view using a combination of two fuzzy techniques, namely, fuzzy relation equation theory and fuzzy graph. Firstly, fuzzy dependence rules are computed from fuzzy relation equations and, secondly, an analysis based on incidence subgraph is performed. The result is a strategic plan automatically generated from the data captured of each enterprise in which the most import variables to be improved are detailed.
\end{abstract}

(C) 2020 The Authors. Published by Atlantis Press SARL.

This is an open access article distributed under the CC BY-NC 4.0 license (http://creativecommons.org/licenses/by-nc/4.0/).

\section{INTRODUCTION}

Nowadays, analytics (data and variable analysis) provide competitive advantages for companies. In fact, Forbes has established that companies that still are not investing heavily in analytics by 2020 probably will not be in business in 2021 [1]. This is due to the great opportunities associated with data and variable analysis for helping companies to get a better understanding of the market and make timely business decisions [2]. At the same time, enterprises need data and variable analysis in order to assess their developments, improvements and achievements. The obtained knowledge will allow them to increase their benefits.

On the other hand, one of the most important problems detected in companies is the necessity of integrating in pursuit of a common goal. The lack of this integration weakens the companies, generating discontent and recurrent problems both internally and externally, creating barriers for the effective fulfillment of the mission and the vision [3,4]. The use of new technologies is also important to increase the efficiency and effectiveness levels in a company.

The integration of a management system for enterprises throughout strategic management models associated with enterprise architecture (EA) is an important contribution of this work since the

*Corresponding author. Email: clrubio@ubiobio.cl current management models do not consider Information Technology (IT) variables in an integrated way. Integration Management System in Enterprises (IMSE) aims this integration from different points of view and, in particular, with the use of IT, throughout strategic management models associated with EA. EA is an important research field in the business sector, which has several approaches: the alignment approach, focuses on interconnect the organization's strategies with IT in order to achieve greater performance [5-7]; the system approach, aims at the holistic representation and coherent distribution of organizational levels in the processes, information systems and technological infrastructure $[8,9]$; the strategic approach, which describes a current stage of the organization through the interconnection level of the processes, the ITs and the strategies, and leads to a future stage or higher level of maturity by using, for example, frameworks, models and tools adapted to different business contexts [10-12]. This model has emerged as a theoretical and practical response to the shortcomings in the field of strategic management with respect to the strategic direction that organizations should take toward the future, taking full advantage of their capabilities, based on a coherent and coordinated relationship between all key and functional processes and external entities [3].

For all these reasons, data and variable analysis in the EA is an important and mandatory tool for companies because it allows them to assess the IMSE, based on EA and IT. As a consequence, the enterprises will have a supporting decision-making mechanism in 
order to know the main actuations they must perform for increasing their benefits, based on the assessment of EA variables. In this paper, analytics is applied to EA discipline.

Emerging analytics research can be classified into five critical technical areas: data analytics, text analytics, web analytics, network analytics and mobile analytics [2]. AI and machine learning become force multipliers for data analytics. One of these techniques is the well-known path analysis (PA) paradigm which aims to asses theoretical models in which a set of dependencies relations between variables is proposed. A new mechanism based on fuzzy logic for assessing a strategic management model is proposed in this paper. This method is based on five phases:

1. Definition of variables. In this phase, the main variables together with the relations among them are established and obtained from a study of the EA literature and prestigious EA experts.

2. Stages and dependences among the variables. In this phase the different variables in EA are analyzed from a theoretical point of view, in order to provide the IMSE model. As a consequence, diverse cause-effect relations, existing among the introduced EA variables, are established. The considered variables are grouped in three stages (identified with 5, 12 and 5 variables each one) by taking into account the strategic management processes presented in an organization.

3. Collect and store observations. Particular values for each variable are obtained from a questionnaire which is answered by a set of experts. The questionnaire is based on a checklist with options between 0 and 1 . The checklist is designed by taking into account theoretical analysis of variables for EA and the semantic relations between variables and the correlations established between them (see Example 1).

4. Fuzzy decision rules analysis. In this phase, dependences are represented as fuzzy decision rules, in which the weights are unknown. In order to compute that weights, different equations arise and so, fuzzy relation equations (FREs) theory [13] is applied to solve them [14-16]. The weight of each rule shows the truth value of the rule, that is, the (observed) real relationship between the variables in the rule. Hence, we can check whether the theoretical dependence is supported by the observations and if this relation should be changed or removed.

5. Priority strategy based on the incidence graph. The development of this set of fuzzy dependence rules also provides a variable prioritization mechanism, that is, offers the most important variables to be improved when the EA needs improvements. In order to do that, a procedure for computing a priority value to each variable, based on the incidence of the variable in the rest of variables of the stage is performed. Specifically, for each variable, all directed paths from this to a final variable will be generated and its associated degree will be computed.

This method has been applied to different Cuban enterprises obtaining good results and providing an effective mechanism to improve the IMSE [17]. This paper aims to strengthen the theoretical study from the observed data of the different considered companies. In order to have a set of heterogeneous data, companies from diverse sectors have been taken into account, such as construction, biopharmaceutical, communications, real estate and tourism sector. If the company has a limited budget to increase the IMSE, with this mechanism the company can focus its efforts on the prioritized variables, whose enhancement also increases other important variables, optimizing the resources and increasing the benefits.

The structure of the paper is as follows: Section 2 recalls the main notions of FREs, Section 3 presents the strategic management model with an EA approach for the IMSE and also details the cause-effect relations (variables grouped in three stages), relations-cause, effects-among them and the definition of rules by using logic implications. In Section 4 dependencies analysis between variables based on fuzzy logic is explained. In Section 5 an evaluation by using priority strategy based on incidence graphs is performed. Finally, diverse conclusions and prospects for future work are included.

\section{FUZZY RELATION EQUATIONS}

The FREs were introduced by E. Sanchez [18] as a mathematical tool based on the composition of fuzzy relations and focused on solving medical problems. From its introduction, FRE has been developed in theoretical and practical aspects. For instance, FRE has also been considered in approximate reasoning, automatic control or decision-making.

Given a set $L$ and an ordering relation $\preceq$, the pair $(L, \preceq)$ forms a complete lattice if for each subset of $L$ there is the minimum of the upper bounds, called supremum, and the maximum of the lower bounds, called infimum. A complete lattice has a minimum and a maximum element, which we will denote as 0 and 1, respectively. Given a set $V$, the ordering $\preceq$ induces a partial order on the fuzzy subsets of $V$, that is, in the set $L^{V}=\{S \mid S: V \rightarrow L\}$. This order is define for each pair of fuzzy subsets $S, S^{\prime} \in L^{V}$, as $S \preceq S^{\prime}$ if and only if $S(v) \preceq S^{\prime}(v)$, for all $v \in V$. The pair $\left(L^{V}, \preceq\right)$ also is a complete lattice.

Different algebraic structures have been considered to define the composition of fuzzy relations. For example, the first composition considered the maximum and the minimum on the unit interval [18]. Recently, more flexible operators have been considered $[15,16,19]$, which have a better adjustment to real cases. For example, the structure considered in [16] will be recalled in this section to be used later.

Given a complete lattice $(L, \preceq)$, an adjoint pair $(\odot, \leftarrow)$ is a pair of mappings $\odot: L \times L \rightarrow L$ and $\leftarrow: L \times L \rightarrow L$, such that satisfy the adjoint property, that is,

$$
x \odot y \preceq z \quad \text { iff } \quad y \preceq z \leftarrow x
$$

for all $x, y, z \in L$. Note that this property is equivalent to $\odot$ preserves the supreme in the second argument: $x \odot \bigvee\{y \mid y \in Y\}=$ $\bigvee\{x \odot y \mid y \in Y\}$, for all $Y \subseteq L$.

Given an adjoint pair $(\odot, \leftarrow)$, a FRE is given by

$$
R \circ X=T
$$

where $R: U \times V \rightarrow L, T: U \times W \rightarrow L$ are known fuzzy relations, $X: V \times W \rightarrow L$ is unknown and $R \circ X$ is defined for each $u \in U, w \in$ $W$, as 


$$
(R \circ X)(u, w)=\bigvee\{R(u, v) \odot X(v, u) \mid v \in V\}
$$

The solvability of these equations was also studied in [16], obtaining that a FRE $R \circ X=T$ has a solution if and only if

$$
(R \Rightarrow T)(v, w)=\bigwedge\{T(u, w) \leftarrow R(u, v) \mid v \in V\}
$$

is a solution and, in that case, it is the maximum solution. When the equation is not solvable, an approximation can be computed [20]. In this last paper, two procedures for computing approximate solutions were introduced and justified, which were called conservative/pessimistic approximation and optimistic approximation. In this article we will consider the former one, which is the greatest solution of the next inequality:

$$
R \circ X \leqslant T
$$

In the study presented in this paper, for illustrating the given procedures, we will consider the lineal lattice $L=[0,1]$, and the Łukasiewicz conjunction and its residuated implication, as adjoint pair: $x \odot y=\max \{0, x+y-1\}$ and $z \leftarrow y=\min \{1,1-y+z\}$, for all $x, y, z \in[0,1]$. Notice that other algebraic structure can be considered for the computations. In the future, the comparison among the use of different structures will be analyzed.

\section{EA TO STRATEGIC MANAGEMENT}

The Strategic Management model focuses on the EA to get a complete Integration Management System in the Enterprise (SMEAIMSE), considered in this paper, is based on IMSE and aims to improve the level of integration of the management system of the enterprise by using the variables of EA and strategic management.

This model is based on the main ISDE approaches: strategic, clientoriented and processes [21], together with a new approach based on EA. The main properties are the following:

- Integration. All management elements of the EA are analyzed in order to determinate which of them are important for the external and internal relationships.

- Teamwork. The model is implemented by a team of experts, which is leaded by the manager of the company, together with the personal involved in the different stages of the processes.

- Predictability. It offers tools for making preventive and flexible decisions within the management system.

The implementation of the model initially requires compliance with several fundamental premises:

1. The processes and flows of the generated information must be defined and identified.

2. The senior managers in the company must feel committed with the proposed changes.

3. The company must have at least one defined strategic plan and work according to the goals reflected in it.

4. The IT must be involved in the strategic plan. IT must be a key element in the strategic plan and its involvement and development must be assessment.

\subsection{Design of the SMEA-IMSE}

Now, we will present the main variables of the EA we have detected, and the relations among them, which have been obtained from a study of the EA literature and prestigious EA experts [17,21]. As a consequence, diverse cause-effect relations, existing among the introduced EA variables, have been established.

The considered variables are grouped in three stages by taking into account the strategic management processes presented in an organization. The relation between EA and Strategic Management has been discussed in previous papers $[17,22]$. For each stage two steps will be carried out:

1. Definition of variables and cause-effect relations among them.

2. Quantification of them and definition of the rules.

\subsubsection{Stage 1}

Process-Based Strategic Design (PSD) is the first stage and is focused on determining the strategic direction of the organization, taking into account mainly the results of the integration level of the enterprise management system and the internal and external strategic diagnosis. The variables involved in this phase are

- Strategic Team (ST, $a_{1}$ ) focuses on the work of management and specialists toward the implementation of the strategic direction, using IT.

- Communication among Stakeholders (CS, $\left.a_{2}\right)$ analyzes the communication relationships between internal and external stakeholders throughout IT or other channels for adding value to the key processes.

- Strategic Project (SP, $\left.a_{3}\right)$ defines or redefines the strategic plan: mission, vision, policies, strategic objectives and measurement criteria, values and organizational competencies.

- Diagnosis, Design and Redesign of the Key Processes (DDRKP, $a_{4}$ ) aims to diagnose, design and/or redesign the key processes and the relationships that increase the value added in products and services.

- Diagnosis, Design and Redesign of the Functional Processes (DDRFP, $a_{5}$ ) whose objective is to diagnose, design and/or redesign the functional processes and their relationships that ensure the functioning of the key processes.

From these variables the theoretical study determines diverse causeeffect relations among them (see Figure 1), which dependences are represented as follows:

1. $a_{2}, a_{3} \rightarrow a_{1}$

2. $a_{3} \rightarrow a_{2}$

3. $a_{1}, a_{2}, a_{3}, a_{5} \rightarrow a_{4}$

4. $a_{1}, a_{2}, a_{3} \rightarrow a_{5}$

For example, the first dependence should be read as follows: $C S$ and $S P$ have a direct effect on $S T$, in this case, we would say that the Communication among the Stakeholders, and the Strategic Project affect to the Strategic Team. 


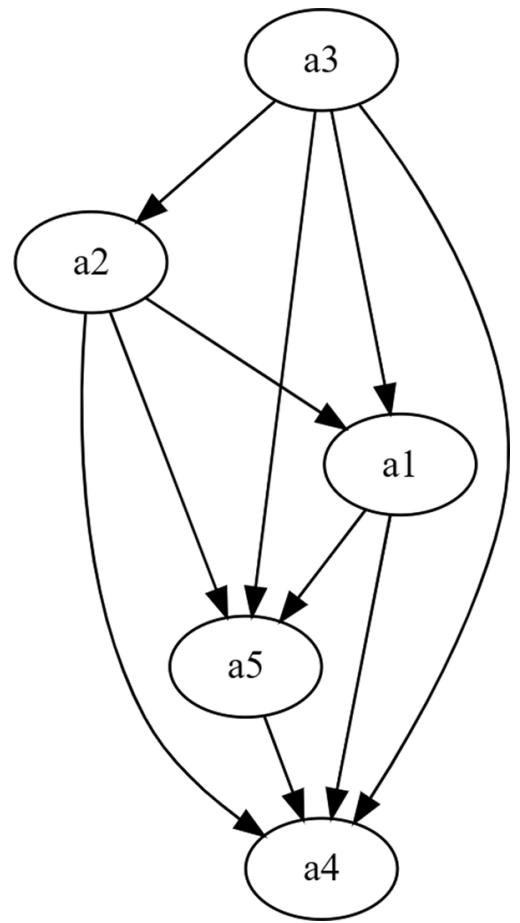

Figure 1 Diagram established between the variables involved at Stage 1.

\subsubsection{Stage 2}

Strategic Design based on the Approach of the EA. This second stage aims to provide solutions based on EA and focusing on the key, strategic and low performance support processes within the corporate system. The variables involved in this stage are

- Technological Vigilance (TV, $\left.b_{1}\right)$ which goal is to identify needs to design, implement and control surveillance actions in the key and functional processes and their relationships.

- Management and Automation of processes (MA, $b_{2}$ ) aims to design, implement and control the actions performed for the automation of processes according to the enterprise needs.

- Response Capacity (RC, $b_{3}$ ) try to develop the response capacity of the processes when environmental changes arise, mainly using IT.

- Management of Relevant Information in processes (MRI, $b_{4}$ ) aims to identify, select, use and control the relevant information in the processes and their relationships through IT.

- Information Security (IS, $b_{5}$ ) is focused on developing the necessary capacities to protect the relevant information of the processes and their relationships.

- Integration of Information for strategic decision-making (II, $b_{6}$ ) aims the integration of relevant information in the processes and their relationships through IT to improve the decision-making.

- Structure of IT Applications $\left(\mathbf{S A}, b_{7}\right)$ purposes to evaluate the design and the implementation of softwares in the functional and key processes.
- Interoperability of IT Applications (IA, $b_{8}$ ) try to evaluate the interoperability and transversal integration of the softwares in the processes and their relations.

- Exploitation of IT Applications in Key processes (EAK, $b_{9}$ ) designs activities to take full advantage of existing softwares in the key processes.

- Investments in Technological Infrastructure (ITI, $b_{10}$ ) targets to provide expert with activities that allow investments in feasible technological infrastructure.

- Exploitation of Technological Infrastructure (ETI, $b_{11}$ ) makes actions to take full advantage of the existing technological infrastructure of softwares.

- Integration Technological infrastructure and IT Applications (ITA, $b_{12}$ ) is focused on integrating the technological infrastructure platforms and the softwares of the key and functional processes, and their relationships.

In this stage, the established cause-effect relations are the following:

1. $b_{1} \rightarrow b_{2}$

2. $b_{1}, b_{2}, b_{4}, b_{5}, b_{6}, b_{7}, b_{6}, b_{12} \rightarrow b_{3}$

3. $b_{1}, b_{2}, b_{6}, b_{7}, b_{9}, b_{11}, b_{12} \rightarrow b_{4}$

4. $b_{1}, b_{4}, b_{10}, b_{12} \rightarrow b_{5}$

5. $b_{2}, b_{7}, b_{8} \rightarrow b_{6}$

6. $b_{2}, b_{5}, b_{12} \rightarrow b_{7}$

7. $b_{1}, b_{2}, b_{4}, b_{5}, b_{12} \rightarrow b_{8}$

8. $b_{1}, b_{7}, b_{8} \rightarrow b_{9}$

9. $b_{1} \rightarrow b_{10}$

10. $b_{1}, b_{9}, b_{10} \rightarrow b_{11}$

\subsubsection{Stage 3}

Implementation, Control and Supervision is the last stage and aims to optimize the model proposed using the strategic plan and a set of actions based on EA. The variables involved in this stage are

- Leadership ( $\left.\mathbf{L}, c_{1}\right)$ aims to lead the processes of implementation, communication, supervision and control described in the strategic plan.

- Assimilation of Changes by the workers (AC, $\left.c_{2}\right)$ which goal is to implement actions to guarantee the assimilation of the proposed changes in the strategic program.

- Management of Efficiency and Effectiveness Indicators (EEI, $c_{3}$ ) targets to control and evaluate the performance of efficiency and effectiveness indicators in strategic processes and indicators based on the implementation of the strategic program by IT.

- Integration of IT with Strategic Objectives and processes (ISO, $c_{4}$ ) is focused on assessing the level of integration of IT with the strategic objectives and the processes once the strategic program has been implemented. 
- Generation of Value (GV, $c_{5}$ ) measures the effect (added value) of the actions carried out in the processes through the IT once the strategic program has been implemented.

Hence, a total of five variables are considered and the theoretical study has determined the following cause-effect relations:
1. $\quad c_{1}, c_{4} \rightarrow c_{2}$
2. $c_{1}, c_{2}, c_{4} \rightarrow c_{3}$
3. $c_{1} \rightarrow c_{4}$
4. $\quad c_{1}, c_{2}, c_{3}, c_{4} \rightarrow c_{5}$

\subsection{Strategic Technological Capacity and Dataset of Observations}

The strategic TEchnological CApacity (TECA) is the main indicator of the strategic management model SMEA-IMSE, which is associated with a specific company. TECA is defined as the ability to manage the design, implementation and control of a strategic project for the integration of the management system of the considered company, throughout EA variables. TECA is computed from the previous set of variables, grouped in the three phases:

\section{Process-Based Strategic Design}

2. Strategic Design based on the Approach of the EA

3. Implementation, Control and Supervision

For each company to be studied, this indicator is computed from the particular values obtained from a checklist that the experts of the company have filled in and the set of cause-effect relations given above [17,21]. These managers and/or specialists of different real companies have answered different questions in the checklist we have prepared for assessing all variables. The questions have a score (with a value between 1 and 10) and they have been aggregated using the geometric measure for computing the TECA. The details of this checklist and the considered procedure are given in [23,24]. Next, an example of question is presented.

Example 1. For example, for the Strategic Project variable (question 1.3 in the checklist [24], which has been summarized in order to be more concise in this paper), the expert must answer the following question: is the Strategic Projection being developed efficiently by the organization? For what the expert has the following options:

1. Not, it is not being developed (0).

2. Yes, a strategic projection for the organization is carried out. Very few objectives are achieved $(0.1,0.2,0.3)$.

3. Yes, a strategic projection for the organization is carried out. Some objectives are achieved $(0.4,0.5,0.6)$.

4. Yes, a strategic projection for the organization is carried out. Many strategic objectives hold $(0.7,0.8,0.9)$.
5. Yes, a strategic projection for the organization is carried out by using ITs. The strategic projection is updated quarterly, this guarantees the fulfillment of all proposed strategic objectives (1).

The following example shows a set of values obtained from a checklist that an expert fills. These values are stored in a dataset of observations, which will be fundamental for the next step (Section 4).

Example 2. For example, for each stage, an expert will establish a score for company $E_{1}$ by indicating the corresponding values for each variables.

- Stage 1: $\left(a_{1}, 7.56\right),\left(a_{2}, 6.44\right),\left(a_{3}, 8.78\right),\left(a_{4}, 9.00\right)$, $\left(a_{5}, 8.78\right)$.

- Stage 2: $\left(b_{1}, 6.89\right),\left(b_{2}, 6.89\right),\left(b_{3}, 6.56\right),\left(b_{4}, 8.78\right),\left(b_{5}, 6.89\right)$, $\left(b_{6}, 7.22\right),\left(b_{7}, 6.33\right),\left(b_{8}, 5.67\right),\left(b_{9}, 7.11\right),\left(b_{10}, 8.11\right)$, $\left(b_{11}, 7.78\right),\left(b_{12}, 7.22\right)$.

- Stage 3: $\left(c_{1}, 8.00\right),\left(c_{2}, 7.22\right),\left(c_{3}, 7.56\right),\left(c_{4}, 7.00\right),\left(c_{5}, 7.89\right)$.

Therefore, in order to compute an efficient and proper TECA for the companies, it is very important to analyze and assess the causeeffect relations obtained from the literature and experts in order to be sure that they correctly hold in the practical cases. This complementary study is introduced in the Section 4, which will be based on the stored dataset.

\section{COMPUTING FUZZY DEPENDENCE RULES FROM FRE}

The established cause-effect relations existing among the variables, in each stage, have been obtained from a theoretical study of EA literature $[17,21]$, as previously was commented. Hence, in order to complement this study, it is fundamental to consider another mechanism that assess the obtained set of cause-effect relations. The selected mechanism is the application of FREs, due to its relation to decision-making and fuzzy dependence rules [14,20], and that the real data does not form a big dataset.

In this section, the dataset obtained from experts (managers and specialists) of several real companies will be considered in order to compute a truth value (weight) to each crisp cause-effect relation given by the theoretical study of the EA literature. As a consequence, fuzzy dependence rules will be obtained, in which the weight of each rule will show its relevance. This truth value will be computed making use of the FREs and the obtained rules will be compared with the crisp ones.

Specifically, from the crisp dependences, a set of fuzzy dependency rules is obtained. Each rule is formed by a head, a body and its corresponding weight, that is, $\langle A \leftarrow B, \vartheta\rangle$. The head $A$ is a fuzzy term formed by the name of the consequent variable acting as a propositional symbol; on the other hand, the body $B$ is a term formed by the name of the antecedent variable acting as a propositional symbol, that is, $\left\langle\right.$ name_variable ${ }_{i} \leftarrow$ name_variable,$\left.x\right\rangle$. 
In $[14,20]$, the authors proved that, given a set of rules:

$$
\left\langle v \leftarrow v_{1}, x_{1}\right\rangle ; \quad\left\langle v \leftarrow v_{2}, x_{2}\right\rangle ; \quad \ldots ; \quad\left\langle v \leftarrow v_{n}, \quad x_{n}\right\rangle
$$

where $v$ is the effect variable, $v_{1}, v_{2}, \ldots, v_{n}$ the causes and $x_{i}$ is the truth value of the rule $v \leftarrow v_{i}$, for all $i \in\{1, \ldots, n\}$; and different instantiations of the variables $v$ and $v_{i}$ for different companies $E_{1}, \ldots, E_{m}$, then the weights $x_{i}$ can be computed, solving the following system of FREs:

$$
\begin{gathered}
v_{1}\left(E_{1}\right) \odot x_{1} \bigvee \ldots \bigvee v_{n}\left(E_{1}\right) \odot x_{n}=v\left(E_{1}\right) \\
\vdots \\
v_{1}\left(E_{m}\right) \odot x_{1} \bigvee \ldots \bigvee v_{n}\left(E_{m}\right) \odot x_{n}=v\left(E_{m}\right)
\end{gathered}
$$

Specifically, from the instantiations of each (effect and cause) variable given by the checklists of each company, a matrix is obtained. This matrix will be used, together with the set of cause-effect rules established in the theoretical framework, to define a FRE. The solutions of this equation will provide the weights (truth values) of these rules.

Notice that, these weights can be interesting for assessing the validity of such rules and for measuring the plausibility of the data observed in future cases. Moreover, these weights can also offer the possibility of providing a priority among the causes of a certain effect, which is very important when the company wants to solve or improve its productivity and/or strategic management. The computed priority will suggest what cause should be improved (increasing its value) firstly in order to increase the effect more quickly. Therefore, if an effect needs to be improved, this priority provides what modification in the values of the causes influences more in the final value of the effect and so, we can optimize the resources given to boost the effects.

In order to design an heterogeneous real data set, six companies from different sectors, sizes and characteristics, are considered. They have been denoted as $E_{1}, \ldots, E_{6}$. Tables $1-3$ include per stage the observed values for the variables for each company.

Next, the procedure will be illustrated for the rules associated with a given variable. Specifically, the effect $a_{1}$ of Stage 1 will be considered. For that variable, there exists two cause-effect relations with head $a_{1},\left(a_{2} \rightarrow a_{1}, a_{3} \rightarrow a_{1}\right)$. Hence, the observed values for $a_{2}, a_{3}$,

Table 1 Input matrix for Stage 1.

\begin{tabular}{lcccccc}
\hline Variable & $\boldsymbol{E}_{\mathbf{1}}$ & $\boldsymbol{E}_{\mathbf{2}}$ & $\boldsymbol{E}_{\mathbf{3}}$ & $\boldsymbol{E}_{\mathbf{4}}$ & $\boldsymbol{E}_{\mathbf{5}}$ & $\boldsymbol{E}_{\mathbf{6}}$ \\
\hline$a_{\mathbf{1}}$ & 7.56 & 6.26 & 2.25 & 3.17 & 7.81 & 6.22 \\
$a_{\mathbf{2}}$ & 6.44 & 6.26 & 3.91 & 4.80 & 15.64 & 7.60 \\
$a_{3}$ & 8.78 & 7.19 & 4.20 & 5.23 & 7.59 & 7.82 \\
$a_{\mathbf{4}}$ & 9.00 & 6.32 & 3.10 & 5.30 & 7.66 & 8.02 \\
$a_{5}$ & 8.78 & 6.16 & 3.83 & 3.88 & 7.70 & 7.63 \\
\hline
\end{tabular}

Table 2 Input matrix for Stage 3.

\begin{tabular}{lcccccc}
\hline Variable & $\boldsymbol{E}_{\mathbf{1}}$ & $\boldsymbol{E}_{\mathbf{2}}$ & $\boldsymbol{E}_{\mathbf{3}}$ & $\boldsymbol{E}_{\mathbf{4}}$ & $\boldsymbol{E}_{\mathbf{5}}$ & $\boldsymbol{E}_{\mathbf{6}}$ \\
\hline$c_{\mathbf{1}}$ & 8.00 & 6.22 & 4.29 & 3.01 & 7.83 & 7.12 \\
$c_{\mathbf{2}}$ & 7.22 & 7.43 & 4.14 & 4.94 & 6.93 & 4.95 \\
$c_{\mathbf{3}}$ & 7.56 & 5.11 & 3.24 & 3.36 & 6.46 & 5.87 \\
$c_{\mathbf{4}}$ & 7.00 & 5.18 & 2.98 & 3.56 & 6.80 & 5.02 \\
$c_{5}$ & 7.89 & 5.74 & 3.23 & 4.74 & 8.25 & 8.12 \\
\hline
\end{tabular}

and $a_{1}$, in each company, are included in the columns of the following matrix, from which the FRE will be defined:

$$
M=\left(\begin{array}{ccc}
0.644 & 0.878 & 0.756 \\
0.626 & 0.719 & 0.626 \\
0.391 & 0.420 & 0.225 \\
0.481 & 0.523 & 0.317 \\
0.564 & 0.759 & 0.781 \\
0.76 & 0.782 & 0.622
\end{array}\right)
$$

The submatrices $R$ (causes) and $T$ (effect) are obtained from $M$ and the equation $R \circ X=T$ is proposed, where $X$ represents the weight of the fuzzy rules: $\left\langle a_{2} \leftarrow a_{1}, \vartheta_{1}\right\rangle,\left\langle a_{3} \leftarrow a_{1}, \vartheta_{2}\right\rangle$, that is, $X$ is the transposed matrix of $\left(\vartheta_{1}, \vartheta_{2}\right)$, that is, we need to solve the equation:

$$
\left(\begin{array}{cc}
0.644 & 0.878 \\
0.626 & 0.719 \\
0.391 & 0.42 \\
0.481 & 0.523 \\
0.564 & 0.759 \\
0.76 & 0.782
\end{array}\right) \circ\left(\begin{array}{l}
\vartheta_{1} \\
\vartheta_{2}
\end{array}\right)=\left(\begin{array}{l}
0.756 \\
0.626 \\
0.225 \\
0.317 \\
0.781 \\
0.622
\end{array}\right)
$$

From the theory of FREs, we know that, if $R \Rightarrow T$ is a solution of the equation $R \circ X=T$, then it is the greatest solution. If it is not a solution, then the equation $R \circ X=T$ is not solvable. In this particular case, we obtain the following strict inequality:

$$
R \circ(R \Rightarrow T)=\left(\begin{array}{cc}
0.644 & 0.878 \\
0.626 & 0.719 \\
0.391 & 0.42 \\
0.481 & 0.523 \\
0.564 & 0.759 \\
0.76 & 0.782
\end{array}\right) \circ\left(\begin{array}{l}
0.834 \\
0.794
\end{array}\right)=\left(\begin{array}{l}
0.672 \\
0.513 \\
0.225 \\
0.317 \\
0.553 \\
0.594
\end{array}\right)<T
$$

As a consequence, $R \Rightarrow T$ is not a solution of the equation. What we can assert is that it is the maximal solution of inequality: $R \circ X \leqslant T$.

The nonsolvability character of the system can be given by the inherent uncertainty of the answers of the experts, the computation error, etc. Hence, although the equation could have some solution, it is not possible to obtain the exact solution. For that cases, Cornejo et al. introduced in [20] two approximation mechanisms. In this paper, the pessimistic/conservative approximation is adapted to the considered problem.

This optimal approximation considers the values of $R \Rightarrow T$ as lower approximation of the truth values of the rules. Consequently, we

Table 3 Input matrix for Stage 2.

\begin{tabular}{lcccccc}
\hline Variable & $\boldsymbol{E}_{\mathbf{1}}$ & $\boldsymbol{E}_{\mathbf{2}}$ & $\boldsymbol{E}_{\mathbf{3}}$ & $\boldsymbol{E}_{\mathbf{4}}$ & $\boldsymbol{E}_{\mathbf{5}}$ & $\boldsymbol{E}_{\mathbf{6}}$ \\
\hline$b_{1}$ & 6.89 & 6.02 & 3.56 & 3.20 & 5.07 & 7.14 \\
$b_{2}$ & 6.89 & 7.08 & 3.82 & 3.99 & 5.21 & 6.82 \\
$b_{3}$ & 6.56 & 6.55 & 2.36 & 2.70 & 6.81 & 7.78 \\
$b_{4}$ & 8.78 & 7.21 & 3.11 & 3.58 & 6.23 & 7.83 \\
$b_{5}$ & 6.89 & 8.34 & 5.73 & 5.50 & 6.32 & 8.47 \\
$b_{6}$ & 7.22 & 7.18 & 3.64 & 3.79 & 6.96 & 7.84 \\
$b_{7}$ & 8.33 & 6.78 & 4.28 & 4.92 & 7.96 & 7.95 \\
$b_{8}$ & 5.67 & 5.96 & 1.97 & 4.33 & 6.68 & 4.84 \\
$b_{9}$ & 7.11 & 6.54 & 3.56 & 4.03 & 5.64 & 6.64 \\
$b_{10}$ & 8.11 & 7.20 & 3.70 & 4.55 & 6.26 & 4.13 \\
$b_{11}$ & 7.78 & 7.50 & 3.04 & 4.20 & 6.68 & 6.40 \\
$b_{12}$ & 7.22 & 7.69 & 2.42 & 4.55 & 5.82 & 5.38 \\
\hline
\end{tabular}


have for the rules $\left\langle a_{2} \leftarrow a_{1}, \vartheta_{1}\right\rangle,\left\langle a_{3} \leftarrow a_{1}, \vartheta_{2}\right\rangle$, that the truth values $\vartheta_{1}$ and $\vartheta_{2}$ are 0.834 and 0.794 , respectively. That is, we have the rules

$$
\begin{aligned}
& \left\langle a_{1} \leftarrow a_{2}, 0.834\right\rangle \\
& \left\langle a_{1} \leftarrow a_{3}, 0.794\right\rangle
\end{aligned}
$$

Therefore, since these true values are high, we can say that the truth values obtained from the application of FRE to the data provided by experts are very close to those established from the theoretical point of view.

Since these truth values show how the cause variables increase the performance of the EA, another important consequence of the computation of these weights, as we previously commented, is that a priority among the variables can be given to improve the TECA. For example, if the manager of the company wants to improve the value of the effect variable $a_{1}$, (s)he can prioritize the development of different activities to improve the variable cause $a_{2}$, whose weight in the cause-effect relation is greater than the one related to the variable (cause) $a_{3}$.

The procedure is applied to every variable in every stage and all the weights are computed. The complete list of weighted rules are given in Tables 4 and 5. These weights complement the diagram (direct graph) in Figures 1-3. The obtained weighted directed graphs are presented in Figures 4-6, respectively.
These graphs provide very interesting information, for example, we can extract from them what variables are more causes and what variables are more effects. For example, in Stage 1, the variable with less incidence in the rest is the variable $a_{4}$ (Diagnosis, Design and Redesign of the Key Processes), which is an effect variable in all cases, this kind of variables will be called target variable. On the other hand, the variable that influences in the rest is the variable $a_{3}$, and no variable influences in it. Hence, $a_{3}$ is a cause variable in all cases, which will be called source variable.

Hence, if for a particular company, the value of the variable $a_{3}$ is not very good and we would like to improve its value, we can only improve this variable by itself, proposing activities to directly improve this variable, since no other variable impacts in it. However, with respect to $a_{4}$, if we improve any of the variables, the value of this target variable will increase. Moreover, these weighted directed graphs quantify how these impacts are given. For example, $a_{3}$ affects to the variables $a_{1}, a_{2}, a_{4}, a_{5}$ with the following degrees: $0.794,0.766,0.89$ and 0.865 , respectively.

Concerning the rest of variables, we can believe that the second most important variable is $a_{2}$ which affects to the variables $a_{1}, a_{4}, a_{5}$ with degrees $0.834,0.919$, and 0.907 , respectively. The third one is $a_{1}$, which is affecting to $a_{4}, a_{5}$ with $0.985,0.989$ respectively. Finally $a_{5}$ affects $a_{4}$ with 0.927 . This ordering can offer a global priority among the variables in order to know what is the most important to be improved in a particular case of a company. Another interesting
Table 4 Fuzzy dependencies rules for Stages 1 and 3.

\begin{tabular}{cc}
\hline Stage $\mathbf{1}$ & Stage 3 \\
\hline$a_{1} \leftarrow a_{2}, 0.834$ & $c_{2} \leftarrow c_{1}, 0.783$ \\
$a_{1} \leftarrow a_{3}, 0.794$ & $c_{2} \leftarrow c_{4}, 0.993$ \\
$a_{2} \leftarrow a_{3}, 0.766$ & $c_{3} \leftarrow c_{1}, 0.863$ \\
$a_{4} \leftarrow a_{1}, 0.985$ & $c_{3} \leftarrow c_{2}, 0.768$ \\
$a_{4} \leftarrow a_{2}, 0.919$ & $c_{3} \leftarrow c_{4}, 0.966$ \\
$a_{4} \leftarrow a_{3}, 0.890$ & $c_{4} \leftarrow c_{1}, 0.790$ \\
$a_{4} \leftarrow a_{5}, 0.927$ & $c_{5} \leftarrow c_{1}, 0.894$ \\
$a_{5} \leftarrow a_{1}, 0.989$ & $c_{5} \leftarrow c_{2}, 0.831$ \\
$a_{5} \leftarrow a_{2}, 0.907$ & $c_{5} \leftarrow c_{3}, 0.999$ \\
$a_{5} \leftarrow a_{3}, 0.865$ & $c_{5} \leftarrow c_{4}, 1.0$ \\
\hline
\end{tabular}

Table 5 Fuzzy dependencies rules for Stage 2.

\begin{tabular}{cl}
\hline Stage 2 & Stage 2 (cont.) \\
\hline$b_{3} \leftarrow b_{1}, 0.880$ & $b_{6} \leftarrow b_{2}, 0.980$ \\
$b_{3} \leftarrow b_{2}, 0.854$ & $b_{6} \leftarrow b_{7}, 0.887$ \\
$b_{3} \leftarrow b_{4}, 0.778$ & $b_{6} \leftarrow b_{8}, 0.946$ \\
$b_{3} \leftarrow b_{5}, 0.663$ & $b_{7} \leftarrow b_{2}, 0.970$ \\
$b_{3} \leftarrow b_{6}, 0.872$ & $b_{7} \leftarrow b_{5}, 0.844$ \\
$b_{3} \leftarrow b_{7}, 0.778$ & $b_{7} \leftarrow b_{12}, 0.909$ \\
$b_{3} \leftarrow b_{8}, 0.837$ & $b_{8} \leftarrow b_{1}, 0.770$ \\
$b_{3} \leftarrow b_{12}, 0.815$ & $b_{8} \leftarrow b_{2}, 0.802$ \\
$b_{4} \leftarrow b_{1}, 0.955$ & $b_{8} \leftarrow b_{4}, 0.689$ \\
$b_{4} \leftarrow b_{2}, 0.929$ & $b_{8} \leftarrow b_{5}, 0.624$ \\
$b_{4} \leftarrow b_{6}, 0.927$ & $b_{8} \leftarrow b_{12}, 0.827$ \\
$b_{4} \leftarrow b_{7}, 0.827$ & $b_{9} \leftarrow b_{1}, 0.950$ \\
$b_{4} \leftarrow b_{9}, 0.955$ & $b_{9} \leftarrow b_{7}, 0.768$ \\
$b_{4} \leftarrow b_{11}, 0.938$ & $b_{9} \leftarrow b_{8}, 0.896$ \\
$b_{4} \leftarrow b_{12}, 0.903$ & $b_{10} \leftarrow b_{1}, 0.699$ \\
$b_{5} \leftarrow b_{1}, 1.000$ & $b_{11} \leftarrow b_{1}, 0.926$ \\
$b_{5} \leftarrow b_{4}, 0.811$ & $b_{11} \leftarrow b_{9}, 0.948$ \\
$b_{5} \leftarrow b_{1} 0,0.878$ & $b_{11} \leftarrow b_{10}, 0.934$ \\
$b_{5} \leftarrow b_{12}, 0.967$ & \\
\hline
\end{tabular}

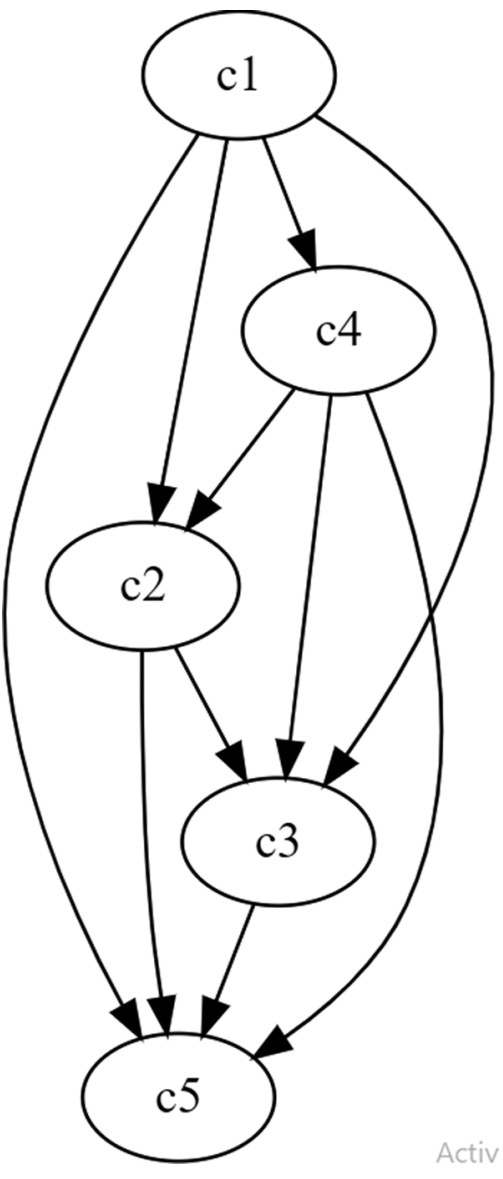

Figure 2 Relations established between the variables involved in Stage 3 . 


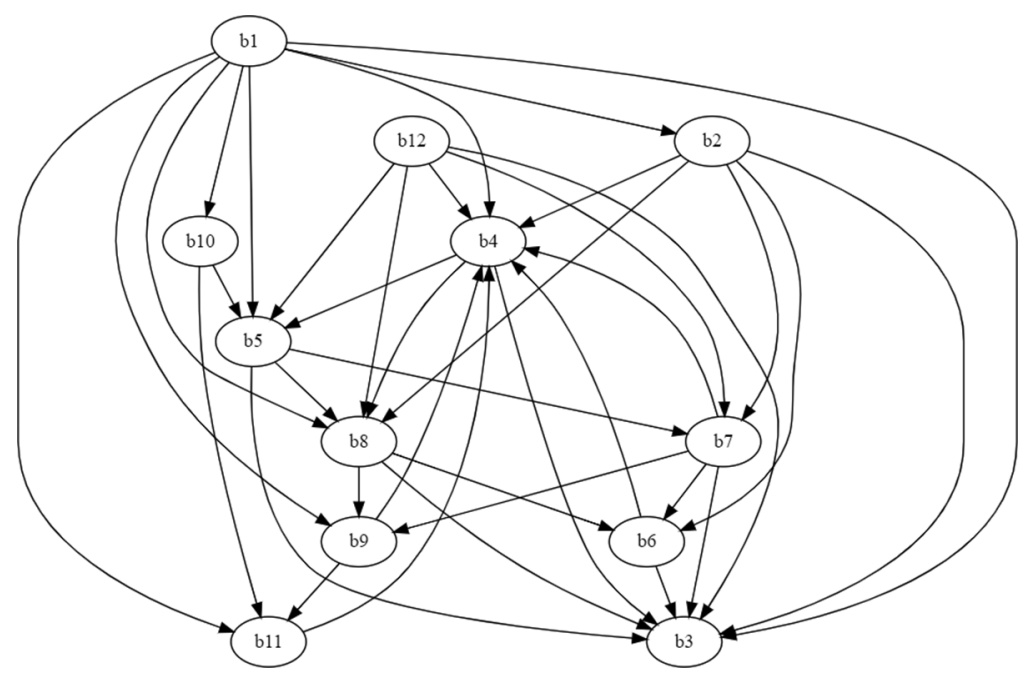

Figure 3 Relationship between the variables of Stage 2.

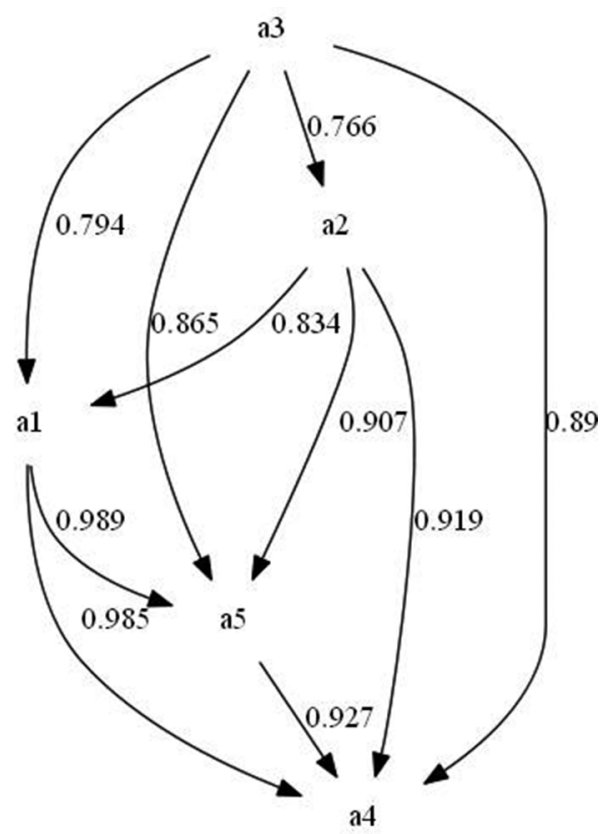

Figure 4 Relations established between the variables involved in Stage 1 and the weights computed by each one of them.

ordering is considering the average of the weights, in this case, for example, $a_{1}$ will be better than $a_{2}$.

Stage 3 also involves few variables and relations and similar strategies of priority can be established. We can see that the variable with less incidence in the rest is $c_{5}$ and the one with more incidence in the rest is $c_{1}$. In addition, considering the weights, we have that the most important variable can be $c_{1}$, which affects to $c_{2}, c_{3}, c_{4}, c_{5}$, with degrees: $0.783,0.863,0.79$ and 0.894 , respectively. The second one can be $c_{4}$ affecting to $c_{2}, c_{3}, c_{5}$ with weights $0.993,0.966$ and 1.0, respectively. The third variable is $c_{2}$, which impacts to $c_{3}, c_{5}$ with weights $0.768,0.831$, respectively, and finally $c_{3}$ affects to $c_{5}$ with weight 0.999 .

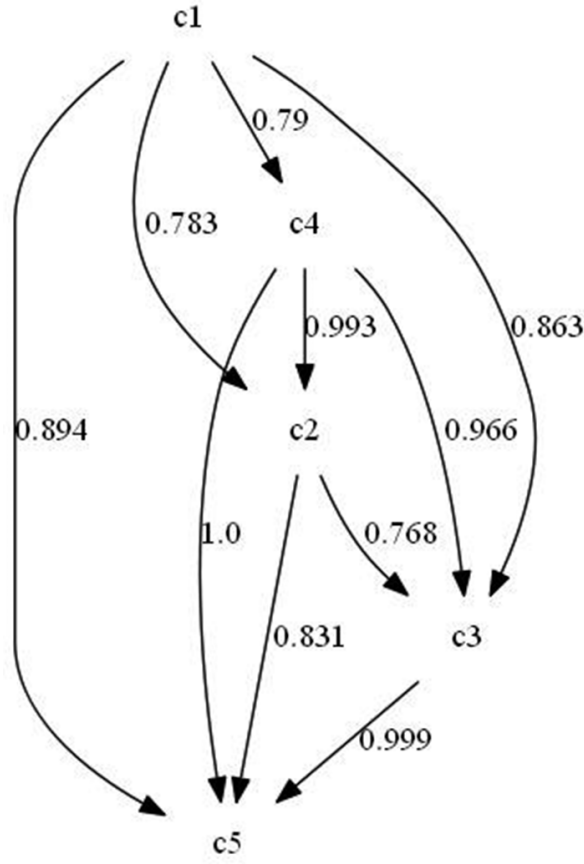

Figure 5 Relations established between the variables involved in Stage 3 and the weights computed by each one of them.
The relations established between the variables involved in Stage 2 are more complex and the computed weights also provide experts useful information about the cause-effect relations. As it can be observed, the variable with less incidence in the rest is the target (cause) variable $b_{3}$ and the one with more impact to the rest is the source variable (effect) $b_{1}$. This variable affects to $b_{2}, b_{3}, b_{4}, b_{5}, b_{9}, b_{10}, b_{11}$ with the following degrees: $0.968,0.84$, $1.0,0.95,0.699$, and 0.926 respectively. We can believe that the other source variable $b_{12}$ is the second variable with more impact to the rest, but variable $b_{2}$ also impacts to 5 variables, what is more important or determinant to be improved? Maybe, the sum of the weights of the variables in which they affect can give us the answer. 


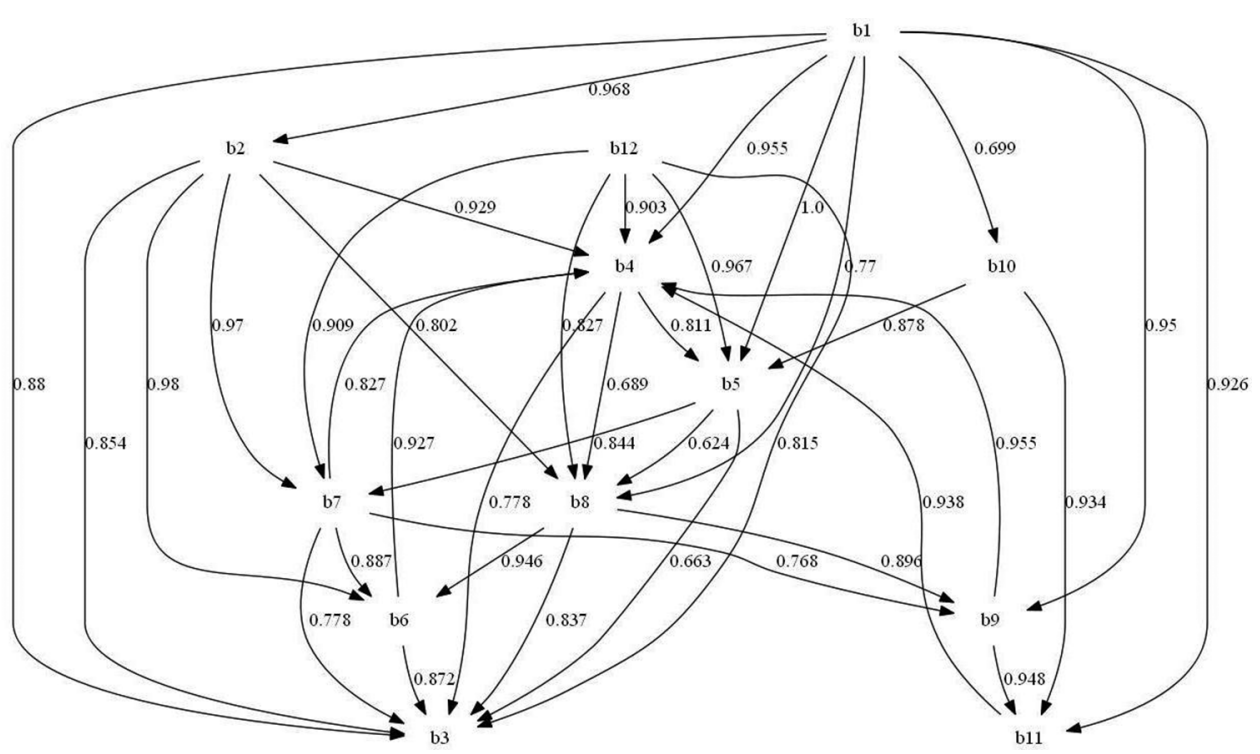

Figure 6 Relations established between the variables involved in Stage 2 and the weights computed by each one of them.

However, the direct influence of each variable to the neighbors variables is not the unique influence in the stage. For example, $b_{2}$ also impacts to $b_{9}$ through $b_{8}$, and to $b_{11}$, through $b_{9}$, etc. Hence, in order to give a representative and effective priority of the variables it is also important to consider all the incidence graph for each variable. This will be studied in the following section.

\section{PRIORITY STRATEGY BASED ON THE INCIDENCE GRAPH}

This section will introduce a procedure for computing a priority value to each variable, based on the incidence of the variable in the rest of variables of the stage. Specifically, for each variable, all directed paths from this to a final variable are generated and its associated degree is computed. The first step will be to compute the local impact of every vertex.

\subsection{Local Impact of Vertices}

For correcting the possible deficiencies detected by the indicator TECA for a particular company, it is important to know what variable must be improved and it is also fundamental to know the incidence of each variable in it. Therefore, we also need to know the incidence of each variable in the rest of variables of every stage. Before formally introducing this notion, we need the following two definitions.

Definition 1. Given a set $V$ (of vertices) and a subset (of weighted edges) $E \subseteq V \times V \times[0,1]$, the pair $(V, E)$ is called weighted directed graph. A weighted directed path from a vertex $v_{0} \in V$ to another vertex $v_{n} \in V$ is a list: where $\left(v_{i-1}, v_{i}, x_{i-1 i}\right) \in E$, for all $i \in\{1, \ldots, n\}$, and it is denoted as Path $\left(v_{0}, v_{n}\right)$. The variables $v_{0}$ and $v_{n}$ are called source and target of the path, respectively.

From the weights considered in a path, a degree associated with the path can be computed.

Definition 2. Given a weighted directed graph $(V, E)$, a triangular norm $(\mathrm{t}$-norm $) \&:[0,1] \times[0,1] \rightarrow[0,1]$, and the weighted directed path:

$$
\operatorname{Path}\left(v_{0}, v_{n}\right)=v_{0} x_{01} v_{1} x_{12} v_{2} \ldots v_{n-1} x_{n-1 n} v_{n}
$$

the degree associated with $\operatorname{Path}\left(v_{0}, v_{n}\right)$ is $\vartheta=\&\left(x_{n-1}, \ldots, x_{12}, x_{01}\right)$. The set of all pairs $\left(\operatorname{Path}\left(v_{0}, v_{n}\right), \vartheta\right)$ between two vertices $v_{0}$ and $v_{n}$ is denoted as $\mathrm{WP}\left(v_{0}, v_{n}\right)$.

Hence, the associated degree is obtained applying a t-norm to all the weights in the path. The operator considered for the examples is the Eukasiewicz conjunction, which is the same operator considered in the resolution of the FRE in Section 4. Table 6 shows all paths and associated weights from $a_{3}$ to the rest of variables.

When several paths arise for the same final variable, the maximum degree of the different paths is computed in order to know the real impact from the source variable to the target one.

Definition 3. Given a weighted directed graph $(V, E)$ and $v_{0}, v_{n} \in$ $V$, the impact of a vertex $v_{0}$ into another vertex $v_{n}$ is the set $\operatorname{Impact}\left(v_{0}, v_{n}\right)$ defined as

$\max \left\{\vartheta \in[0,1] \mid\left(\operatorname{Path}\left(v_{0}, v_{n}\right), \vartheta\right) \in \mathrm{WP}\left(v_{0}, v_{n}\right)\right\}$

In the computation of the impact, the maximum operator collects the useful information, since it shows the most efficient path to be considered in the computation of the influence between two vertices. For example, given the variable $a_{3}$, there are 4 paths from $a_{3}$ to $a_{5}$, which are detailed in Table 6 , and the maximum weight is given by the path $a_{3} 0.865 a_{5}$. 
Figures 7 and 8 present the different paths with different kind of lines (continue, dash, etc.), from $a_{3}$ to the variables $a_{1}, a_{5}$ and $a_{4}$,

Table 6 Path degrees for the source variable $a_{3}$.

\begin{tabular}{lccc}
\hline Source & Target & Path & Degree \\
\hline a3 & a2 & {$[a 3, a 2]$} & 0.766 \\
a3 & a1 & {$[a 3, a 1]$} & 0.794 \\
a3 & a1 & {$[a 3, a 2, a 1]$} & 0.600 \\
a3 & a5 & {$[a 3, a 5]$} & 0.865 \\
a3 & a5 & {$[a 3, a 2, a 5]$} & 0.673 \\
a3 & a5 & {$[a 3, a 1, a 5]$} & 0.782 \\
a3 & a5 & {$[a 3, a 2, a 1, a 5]$} & 0.589 \\
a3 & a4 & {$[a 3, a 4]$} & 0.89 \\
a3 & $\mathrm{a} 4$ & {$[a 3, a 2, a 4]$} & 0.685 \\
a3 & a4 & {$[a 3, a 1, a 4]$} & 0.778 \\
a3 & a4 & {$[a 3, a 2, a 1, a 4]$} & 0.585 \\
a3 & a4 & {$[a 3, a 5, a 4]$} & 0.792 \\
a3 & a4 & {$[a 3, a 2, a 5, a 4]$} & 0.600 \\
a3 & a4 & {$[a 3, a 2, a 1, a 5, a 4]$} & 0.516 \\
\hline
\end{tabular}

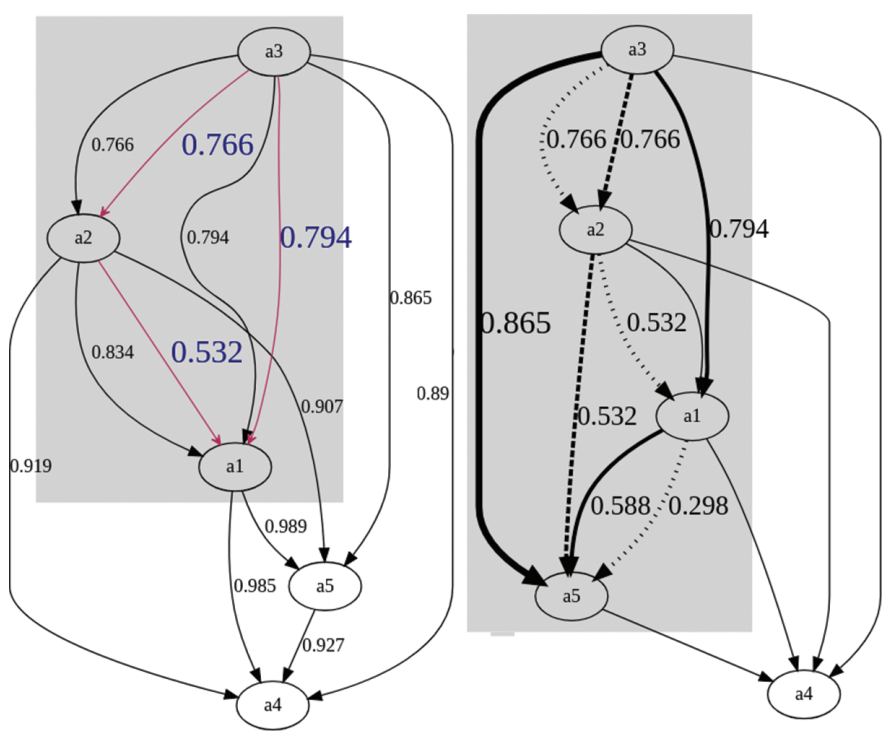

Figure 7 Incidence subgraph of the variable (right).

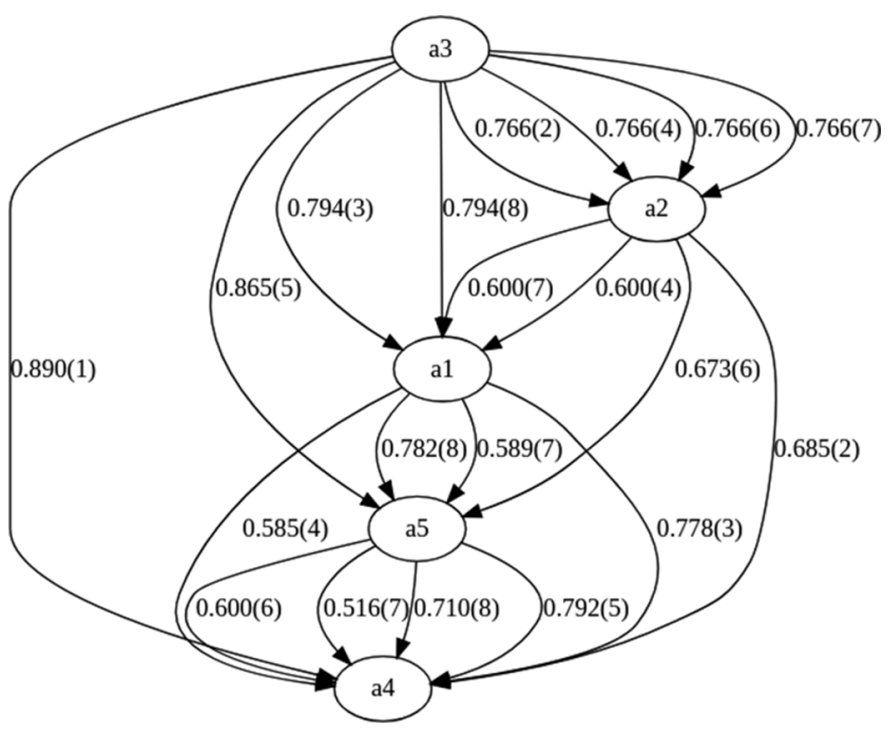

Figure 8 Incidence subgraph of the variable. respectively, and how the computation of the paths is obtained. For example, in the diagram on the left of Figure 7 the red arrow from $a_{3}$ to $a_{2}$ shows the obtained weight, which is the same as the weighted edge, since only one edge is considered in the computation. Now, the red arrow from $a_{2}$ to $a_{1}$ has as weight 0.6 , which is obtained from the weight in the previous red arrow 0.766 and the weight of the corresponding edge 0.834 , applying the Łukasiewicz conjunctor, that is, $0.6=\&_{£}(0.766,0.834)$. In Figure 8 , the weights in the edges are given together with a number between brackets, which shows the number of the path from $a_{3}$ to $a_{4}$. For example, path 7 begins in the weight edge connecting $a_{3}$ and $a_{2}$, with weight 0.766 , which is denoted as $\left(a_{3}, a_{2}, 0.766\right)$. Then, it considers the edge ( $\left.a_{2}, a_{1}, 0.834\right)$, obtaining the weight 0.6 , then it take into consideration the edge $\left(a_{1}, a_{5}, 0.989\right)$, and 0.589 is computed. Finally, the edge $\left(a_{5}, a_{4}, 0.927\right)$ provides the final value 0.516 .

From these paths, the maximum value is computed for each pair $a_{3}$, $a_{i}$, with $i \in\{1,2,4,5\}$, which show the appropriate impact of $a_{3}$ to the other variable. This computation is presented in Table 7.

Notice that $\operatorname{Impact}\left(v_{0}, v_{n}\right)$ is directly related to the computation of the least fixed point of the immediate consequences operator of the program composed of the weighted rules of every stage [25], as we will show in Section 5.2. This relation also shows that $\operatorname{Impact}\left(v_{0}, v_{n}\right)$ is appropriately defined.

\subsection{Relationship with the Fixed-Point Semantics}

We need to take into consideration that the set of rules given for each stage and detailed in Tables 4 and 5 are fuzzy logic programs [26-28], which can be denoted as $\mathbb{P}_{1}, \mathbb{P}_{2}$ and $\mathbb{P}_{3}$. Moreover, their associated directed graph given in Figures $4-6$ will be denoted as $\left(x_{\mathbb{P}_{1}}, E_{\mathbb{P}_{1}}\right),\left(x_{\mathbb{P}_{2}}, E_{\mathbb{P}_{2}}\right)$ and $\left(x_{\mathbb{P}_{3}}, E_{\mathbb{P}_{3}}\right)$, respectively.

Therefore, in order to obtain deductions and consequences from observed values (facts) an operational semantics procedure can be applied, such as the one given by the fixed point semantics [25,29], which is based on the immediate consequences operator. Next, only the definition of this operator will be recalled, the details of this theory can be checked in [26,30].

Definition 4. Let $(\odot, \leftarrow)$ be an adjoint pair on the unit interval, $\mathbb{P}$ a fuzzy logic program and $\Pi_{\mathbb{P}}$ the set of propositional symbols in $\mathbb{P}$, the immediate consequences operator $T_{\mathbb{P}}$ maps interpretations to interpretations, and for an interpretation $I: \Pi_{\mathbb{P}} \rightarrow L$ and an $A \in \mathbb{P}$ is defined as

$$
T_{\mathbb{P}}(I)(A)=\sup \{\vartheta \odot \hat{I}(\mathcal{B}) \mid\langle A \leftarrow \mathcal{B}, \vartheta\rangle \in \mathbb{P}\}
$$

From this operator, it is well-known that the consequences arise from the least model of the program, which coincides with the least

Table 7 Fuzzy incidence of $a_{3}$ to the rest of variables in Stage 1 .

\begin{tabular}{lccc}
\hline Source & Target & Path & Impact of $\boldsymbol{a}_{\mathbf{3}}$ to $\boldsymbol{a}_{\boldsymbol{i}}$ \\
\hline a3 & a2 & {$[a 3, a 2]$} & 0.766 \\
a3 & a1 & {$[a 3, a 1]$} & 0.794 \\
a3 & a5 & {$[a 3, a 5]$} & 0.865 \\
a3 & a4 & {$[a 3, a 4]$} & 0.89 \\
\hline
\end{tabular}


fixed point of $T_{\mathbb{P}}$, denoted as $\operatorname{lfp}\left(T_{\mathbb{P}}\right)$ and it is obtained iterating the $T_{\mathbb{P}}$ operator from the least interpretation $\Delta$.

Notice that, the vertices of the associated directed graph $\left(x_{\mathbb{P}}, E_{\mathbb{P}}\right)$ of a program $\mathbb{P}$ coincides with the propositional symbols in the program. The following result relates the model of a program $\mathbb{P}$ to the impacts of the vertices of $\left(x_{\mathbb{P}}, E_{\mathbb{P}}\right)$.

Theorem 1. Given an adjoint pair $(\&, \leftarrow)$ on the unit interval, where $\&$ is a $t$-norm, and a fuzzy logic program $\mathbb{P}$, the immediate consequences operator $T_{\mathbb{P}}$ and its associated directed graph $\left(x_{\mathbb{P}}, E_{\mathbb{P}}\right)$, we have that

$$
\operatorname{lfp}\left(T_{\mathbb{P}}\right)\left(v_{n}\right)=\max \left\{\operatorname{Impact}\left(v_{i}, v_{n}\right) \& T_{\mathbb{P}}(\Delta)\left(v_{i}\right) \mid v_{i} \in x_{\mathbb{P}}\right\}
$$

Proof. Given $v_{n} \in x_{\mathbb{P}}$ and $\left\langle v_{n} \leftarrow \mathcal{B}, \vartheta\right\rangle \in \mathbb{P}$, then the value taken into account to compute $T_{\mathbb{P}}^{k}(\Delta)\left(v_{n}\right)$ is $\vartheta \& \widehat{T_{\mathbb{P}}^{k-1}(\Delta)}(\mathcal{B})$. Therefore, given $v_{0} \in x_{\mathbb{P}}$ and a path $\operatorname{Path}\left(v_{0}, v_{n}\right)$ :

$$
v_{0} x_{01} v_{1} x_{12} v_{2} \ldots v_{n-2} x_{n-2 n-1} v_{n-1} x_{n-1} v_{n}
$$

where $\left(v_{i-1}, v_{i}, x_{i-1}\right) \in E_{\mathbb{P}}$, for all $i \in\{1, \ldots, n\}$, we have that the rules in the following list belong to $\mathbb{P}$ :

$$
\begin{aligned}
& \left\langle v n \leftarrow v_{n-1}, x_{n-1}\right\rangle,\left\langle v_{n-1} \leftarrow v_{n-2}, x_{n-2 n-1}\right\rangle, \ldots, \\
& \left\langle v_{2} \leftarrow v_{1}, x_{12}\right\rangle,\left\langle v_{1} \leftarrow v_{0}, x_{01}\right\rangle
\end{aligned}
$$

As a consequence, if $k=n+1$, the following chain of inequalities holds

$$
\begin{aligned}
T_{\mathbb{P}}^{n+1}(\Delta)\left(v_{n}\right) & \geqslant x_{n-1 n} \& \widehat{T_{\mathbb{P}}^{n}(\Delta)}\left(v_{n-1}\right) \\
& \left.\geqslant x_{n-1 n} \&\left(x_{n-2 n-1} \& \widehat{T_{\mathbb{P}}^{n-1}(\Delta)}\right)\left(v_{n-2}\right)\right) \\
& \vdots \\
& \geqslant x_{n-1{ }_{n}} \& \ldots\left(x_{12} \&\left(x_{01} \& \widehat{T_{\mathbb{P}}(\Delta)}\left(v_{0}\right)\right)\right)
\end{aligned}
$$

Thus, by the associativity of \& we obtain that

$$
\operatorname{Impact}\left(v_{i}, v_{n}\right) \& T_{\mathbb{P}}(\Delta)\left(v_{i}\right) \leqslant T_{\mathbb{P}}^{n+1}(\Delta)\left(v_{n}\right)
$$

Since this procedure can be applied to all $v_{i} \in x_{\mathbb{P}}$ and rule with head $v_{i}$, we obtain the equality

$$
\max \left\{\operatorname{Impact}\left(v_{i}, v_{n}\right) \& T_{\mathbb{P}}(\Delta)\left(v_{i}\right) \mid v_{i} \in x_{\mathbb{P}}\right\}=\operatorname{lfp}\left(T_{\mathbb{P}}\right)\left(v_{n}\right)
$$

As a consequence, the computation of the least model on a propositional symbol (vertex) depends on the impacts of the rest of vertices on it. Therefore, this result really justifies that $\operatorname{Impact}\left(v_{0}, v_{n}\right)$ computes the influence of vertex $v_{0}$ to vertex $v_{n}$. Notice also that the unfolding process presented in [27] also accumulates the values as it is computed for $\operatorname{Impact}\left(v_{0}, v_{n}\right)$. The Section 5.3 extents the notion of impact of a vertex to all graph.

\subsection{Global Impact of Vertices}

It is also very interesting to know a global impact indicator of the variables involved in every stage. This indicator will be given from the sum of the appropriate impact to the rest of variables.
Definition 5. Let $(V, E)$ be a weighted directed graph, and the set of all pairs $\left(\operatorname{Path}\left(v_{0}, v_{n}\right), \vartheta\right)$ between two vertices $v_{0}$ and $v_{n}$, $\mathrm{WP}\left(v_{0}, v_{n}\right)$. The fuzzy incidence degree (FID) of the vertex $v_{0}$ into the graph, is

$$
\operatorname{FID}\left(v_{0}\right)=\sum\left\{\operatorname{Impact}\left(v_{0}, v_{n}\right) \mid v_{n} \in V \text { and } \mathrm{WP}\left(v_{0}, v_{n}\right) \neq \varnothing\right\}
$$

Another interesting factor is the "quality" of this influence, how big this influence is. This degree is obtained normalizing the FID, that is, computing the average of the FID with the number of target variables.

Definition 6. Given a weighted directed graph $(V, E)$ and a vertex $v_{0} \in V$. The normalized fuzzy incidence degree (NFID) from $v_{0}$ into $(V, E)$ is

$$
\operatorname{NFID}\left(v_{0}\right)=\frac{\operatorname{FID}\left(v_{0}\right)}{\left|\left\{v_{n} \in V \mid \operatorname{WP}\left(v_{0}, v_{n}\right) \neq \varnothing\right\}\right|}
$$

where $|X|$ computes the cardinal of a set $X$.

These last values/indicators provide fundamental information with respect to the global and normalized impact that a variable has in the rest of variables of the stage. Tables 8-10 show these values for the three stages. These figures also include the crisp priority observed in the previous section, the degrees FID and NFID. These last values provide a global priority (FID), which coincide with the crisp priority in Stages 1 and 3, and improve the priority in Stage 2, and a normalized priority (NFID), which shows a better ordering of the variables to be improved, if we are focused on a specific goal variable, only considering the variables involved in the incidence graph of this fixed variable. For example, if we want to improve variable $a_{4}$, it is more appropriate to improve the variables with the following priority: first of all $a_{1}$, secondly $a_{5}$, then $a_{2}$ and finally $a_{3}$. If we are focused on $c_{3}$ in Stage 3, we need to firstly improve $c_{4}$, then $c_{1}$ and finally $c_{2}$. Notice, that $c_{5}$ is not considered since it does not impact to $c_{3}$, that is, $c_{5}$ is not cause of $c_{3}$.

Table 8 Crisp priority, FID and NFID of Stage 1.

\begin{tabular}{lcll}
\hline Variable & Crisp Priority & FID & NFID \\
\hline a3 & 4 & 3.315 & 0.828 \\
a2 & 3 & 2.66 & 0.886 \\
a1 & 2 & 1.978 & 0.989 \\
a5 & 1 & 0.927 & 0.927 \\
a4 & 0 & 0.0 & 0.0 \\
\hline
\end{tabular}

FID, fuzzy incidence degree; NFID, normalized fuzzy incidence degree.

Table 9 Crisp priority, FID and NFID of Stage 3.

\begin{tabular}{lcll}
\hline Variable & Crisp Priority & FID & NFID \\
\hline c1 & 4 & 3.337 & 0.834 \\
c4 & 3 & 2.985 & 0.995 \\
c2 & 2 & 1.599 & 0.7995 \\
c3 & 1 & 0.999 & 0.999 \\
c5 & 0 & 0.0 & 0.0 \\
\hline
\end{tabular}

FID, fuzzy incidence degree; NFID, normalized fuzzy incidence degree. 
Table 10 Crisp priority, FID and NFID of Stage 2.

\begin{tabular}{lcrc}
\hline Variable & Crisp Priority & FID & NFID \\
\hline b1 & 8 & 10.936 & 0.994 \\
b2 & 5 & 8.780 & 0.975 \\
b12 & 5 & 8.604 & 0.956 \\
b7 & 4 & 6.916 & 0.864 \\
b4 & 3 & 5.555 & 0.793 \\
b8 & 3 & 5.567 & 0.927 \\
b5 & 3 & 3.439 & 0.688 \\
b6 & 2 & 3.215 & 0.803 \\
b9 & 2 & 1.896 & 0.948 \\
b10 & 2 & 1.868 & 0.934 \\
b11 & 1 & 0.875 & 0.875 \\
\hline
\end{tabular}

FID, fuzzy incidence degree; NFID, normalized fuzzy incidence degree.

\section{CONCLUSIONS AND FUTURE WORK}

In this paper, a strategic management model has been proposed which allows companies to evaluate EA variables as an IT management approach and to contribute to the IMSE, which has been the first goal of the paper. For assessing this model, we have used the called strategic TECA indicator which provides organizations with the ability to manage the design, implementation and control of a strategic project for the integration of the management system of the considered company, throughout EA variables. The different variables needed in the design of the EA have been selected, classified and related following a theoretical study.

For each company to be studied, this indicator is computed from the particular values obtained from a check-list that the experts of the company fill in and the set of cause-effect relations given above. In this process, cause-effect relations defined between variables and obtained from the literature and experts are analyzed and evaluated in order to be sure that they correctly hold in the practical cases. Additionally, a method for assessing interrelated variables of EA in a strategic management model based on integration theory of management systems in enterprises has been presented and detailed. It can be seen as a novel cause-effect variable analysis method for Integration Management Systems in Enterprises which employs fuzzy logic techniques: fuzzy dependence rules has been obtained by making use of the FREs; we have shown as these weights can be interesting for assessing the validity of such rules and for measuring the plausibility of the data observed in future cases. In particular, the relations given by the theoretical study have been checked considering fuzzy decision rules, computing the weights of these rules and highlighting the most important relationships. These fuzzy rules have also been fundamental for providing a priority in the variables to be improved when the indicator TECA of the IMSE of the company needs to be improved.

Specifically, we have shown how these weights can also offer the possibility of providing a priority among the causes of a certain effect, which is very important when the company wants to solve or improve its productivity and/or strategic management. Therefore, if an effect needs to be improved, this priority provides what modification in the values of the causes influences more in the final value of the effect and so, we can optimize the resources given to boost the effects.

Thus, FREs, fuzzy rules and incidence graphs have been considered to the second main goal of the paper, that is, providing an effective mechanism to improve the IMSE of the companies.
In the future, other complementary tools will be considered, such as fuzzy logic programming, which can be used for analyzing the approximate solution given by the FRE and the possible loops in the (incidence) graphs. Other interesting tool is Fuzzy Cognitive Maps, since they can also be useful for handling cicyles. Furthermore, since the introduced mechanism is portable, that is, it does not depend on the country of the companies, it will be applied to other real companies in Europe and other continents.

\section{CONFLICT OF INTEREST}

The authors declare no conflicts of interest.

\section{Funding Statement}

Partially supported by the State Research Agency (AEI) and the European Regional Development Fund (FEDER) project TIN2016-76653-P and by the European Cooperation in Science \& Technology (COST) Action CA17124.

\section{ACKNOWLEDGMENTS}

The author would like to thank the anonymous referees for their careful reading of the paper and useful suggestions to clarify this work.

\section{REFERENCES}

[1] D. Newman, Top 10 digital transformation trends for 2020, Forbes, 2019. https://www.forbes.com/sites/danielnewman/ 2019/07/14/top-10-digital-transformation-trends-for-2020/

[2] H. Chen, R.H. Chiang, V.C. Storey, Business intelligence and analytics: from big data to big impact, MIS Q. 36 (2012), 1165-1188.

[3] D. Alfonso-Robaina, Modelo de dirección estratégica para la integración del sistema de dirección de la empresa, PhD Thesis, Universidad Tecnológica de La Habana José Antonio Echeverría, Cuba, 2007.

[4] M. Bugdol, P. Jedinak, Integrated Management Systems, Springer International Publishing, Cham, Switzerland, 2015.

[5] K. Hinkelmann, A. Gerber, D. Karagiannis, B. Thoenssen, A. Van der Merwe, R. Woitsch, A new paradigm for the continuous alignment of business and it: combining enterprise architecture modelling and enterprise ontology, Comput. Indus. 79 (2016), 77-86.

[6] M. Lankhorst, Enterprise Architecture at Work: Modelling, Communication and Analysis, vol. 352, Springer, Berlin, Heidelberg, 2009.

[7] J. Lapalme, Three schools of thought on enterprise architecture, IT Prof. 14 (2011), 37-43.

[8] M. Berrada, B. Bounabat, Business modeling of enterprise architecture based on multi-agent system, Int. J. e-Educ. e-Bus. eManag. e-Learn. 3 (2013), 472.

[9] F.B. Vernadat, Interoperable enterprise systems: principles, concepts, and methods, Ann. Rev. Control. 31 (2007), 137-145.

[10] C. Braun, R. Winter, Integration of it service management into enterprise architecture, in Proceedings of the 2007 ACM Symposium on Applied Computing, ACM, Seoul, Korea, 2007, pp. 12151219. 
[11] P. Johnson, M. Ekstedt, E. Silva, L. Plazaola, Using enterprise architecture for CIO decision-making: on the importance of theory, in Second Annual Conference on Systems Engineering Research, 2004. http://www.diva-portal.org/smash/get/diva2: 509306/FULLTEXT01.pdf

[12] R. Winter, R. Fischer, Essential layers, artifacts, and dependencies of enterprise architecture, in 10th IEEE International Enterprise Distributed Object Computing Conference Workshops (EDOCW'06), IEEE, Hong Kong, China, 2006, pp. 30-30.

[13] Z. Pawlak, Information systems theoretical foundations, Inf. Syst. 6 (1981), 205-218.

[14] J.C. Díaz, J. Medina, Multi-adjoint relation equations: definition, properties and solutions using concept lattices, Inf. Sci. 253 (2013), 100-109.

[15] J.C. Díaz-Moreno, J. Medina, Using concept lattice theory to obtain the set of solutions of multi-adjoint relation equations, Inf. Sci. 266 (2014), 218-225.

[16] J.C. Díaz-Moreno, J. Medina, E. Turunen, Minimal solutions of general fuzzy relation equations on linear carriers. An algebraic characterization, Fuzzy Sets Syst. 311 (2017), 112-123.

[17] A. Malleuve-Martínez, D. Alfonso-Robaina, J. Lavandero-García, Study of elements behavior for integration management system with enterprise architecture approach, Dyna. 84 (2017), 349-355.

[18] E. Sanchez, Resolution of composite fuzzy relation equations, Inf. Control. 30 (1976), 38-48.

[19] J. Medina, Minimal solutions of generalized fuzzy relational equations: clarifications and corrections towards a more flexible setting, Int. J. Approx. Reason. 84 (2017), 33-38.

[20] M.E. Cornejo, J.C. Díaz-Moreno, J. Medina, Multi-adjoint relation equations: a decision support system for fuzzy logic, Int. J. Intell. Syst. 32 (2017), 778-800.
[21] A. Malleuve-Martínez, D. Alfonso-Robaina, J. Lavandero-García, V.C. Ramos-Díaz, Strategic management model with enterprise architecture approach for integration management system in enterprises, Dyna. 85 (2018), 297-305.

[22] D. Simon, K. Fischbach, D. Schoder, Enterprise architecture management and its role in corporate strategic management, Inf. Syst. e-Bus. Manag. 12 (2014), 5-42.

[23] A. Malleuve-Martínez, V.C. Ramos-Díaz, D. Alfonso-Robaina, Integración del sistema de dirección con enfoque de arquitectura empresarial en una empresa de comunicaciones, Ingeniería Ind. 40 (2019), 67-77.

[24] A. Malleuve, D. Alfonso-Robaina, J. Medina, C. Rubio-Manzano, Checklist of model: integration management system in the enterprise (SMEA-IMSE), 2019. https://harmonic.uca.es/eafl/.

[25] M.V. Emden, R. Kowalski, The semantics of predicate logic as a programming language, J. ACM. 23 (1976), 733-742.

[26] C.V. Damásio, L.M. Pereira, Monotonic and residuated logic programs, in: S. Benferhat, P. Besnard (eds.), Symbolic and Quantitative Approaches to Reasoning with Uncertainty, ECSQARU'01, Lecture Notes in Artificial Intelligence, vol. 2143, Springer, Berlin, Heidelberg, Toulouse, France, 2001, pp. 748-759.

[27] P. Julin, G. Moreno, J. Penabad, On fuzzy unfolding: a multiadjoint approach, Fuzzy Sets Syst. 154 (2005), 16-33.

[28] J. Medina, M. Ojeda-Aciego, P. Vojtáś, Similarity-based unification: a multi-adjoint approach, Fuzzy Sets Syst. 146 (2004), 43-62.

[29] J. Lloyd, Foundations of Logic Programming, Springer, Berlin, Heidelberg, 1987.

[30] J. Medina, M. Ojeda-Aciego, P. Vojtáš, Multi-adjoint logic programming with continuous semantics, in: T. Eiter, W. Faber, M. Truszczyński (Eds.), Logic Programming and Nonmotonic Reasoning, vol. 2173, Springer, Berlin, Heidelberg, Vienna, Austria, 2001, pp. 351-364. 